\title{
Decitabine, a DNA methyltransferase inhibitor, induces apoptosis in human leukemia cells through intracellular reactive oxygen species generation
}

\author{
DONG YEOK SHIN ${ }^{1,2}$, YOU-SOO PARK ${ }^{2}$, KWANGMO YANG ${ }^{2}$, GI-YOUNG KIM ${ }^{3}$, \\ WUN-JAE KIM ${ }^{4}$, MIN HO HAN ${ }^{5}$, HO SUNG KANG ${ }^{2}$ and YUNG HYUN CHOI ${ }^{5,6}$
}

\begin{abstract}
${ }^{1}$ Department of Molecular Biology, Busan National University, Busan 609-735; ${ }^{2}$ Dongnam Institute of Radiological and Medicine Sciences, Busan 619-953; ${ }^{3}$ Laboratory of Immunobiology, Department of Marine Life Sciences, Jeju National University, Jeju 690-756; ${ }^{4}$ Department of Urology, College of Medicine, Personalized Tumor Engineering Research Center, Chungbuk National University, Chungbuk 361-763; ${ }^{5}$ Department of Biomaterial Control (BK21 program), Graduate School, Anti-Aging Research Center and Blue-Bio Industry RIC, Dongeui University, Busan 614-714; ${ }^{6}$ Department of Biochemistry, Dongeui University College of Oriental Medicine, Busan 614-052, Republic of Korea
\end{abstract}

Received April 9, 2012; Accepted May 16, 2012

DOI: $10.3892 /$ ijo.2012.1546

\begin{abstract}
The DNA methyltransferase inhibitor decitabine, 5-aza-2'-deoxycytidine, has been found to exert anti-metabolic and anticancer activities when tested against various cultured cancer cells. Furthermore, decitabine has been found to play critical roles in cell cycle arrest and apoptosis in various cancer cell lines; however, these roles are not well understood. In this study, we investigated decitabine for its potential anti-proliferative and apoptotic effects in human leukemia cell lines U937 and HL60. Our results indicated that treatment with decitabine resulted in significantly inhibited cell growth in a concentration- and time-dependent manner by the induction of apoptosis. Decitabine-induced apoptosis in U937 and HL60 cells was correlated with the downregulation of anti-apoptotic Bcl-2, XIAP, cIAP-1 and cIAP-2 protein levels, the cleavage of Bid proteins, the activation of caspases and the collapse of mitochondrial membrane potential (MMP). However, apoptosis induced by decitabine was attenuated by caspase inhibitors, indicating an important role for caspases in decitabine responses. The data further demonstrated that decitabine increased intracellular reactive oxygen species (ROS) generation. Moreover, $\mathrm{N}$-acetyl-L-cysteine, a widely
\end{abstract}

Correspondence to: Dr Ho Sung Kang, Department of Molecular Biology, Busan National University, Busan 609-735, Republic of Korea

E-mail: hspkang@pusan.ac.kr

Dr Yung Hyun Choi, Department of Biochemistry, Dongeui University College of Oriental Medicine, Busan 614-052, Republic of Korea

E-mail: choiyh@deu.ac.kr

Key words: decitabine, leukemia, apoptosis, caspase, reactive oxygen species used ROS scavenger, effectively blocked the decitabine-induced apoptotic effects via inhibition of ROS production and MMP collapse. These observations clearly indicate that decitabineinduced ROS in human leukemia cells are key mediators of MMP collapse, which leads to apoptosis induction followed by caspase activation.

\section{Introduction}

Leukemia, a malignant hematopoietic tumor, is a cancer of the blood or bone marrow characterized by the abnormal proliferation of white blood cells; it ranks sixth in the prevalence of human tumors worldwide (1). Leukemias are classified into two subtypes: acute lymphocytic leukemia originating from lymphocytes in the bone marrow and myelogenous leukemia originating from granulocytes or monocytes $(2,3)$. Treatment of leukemia is difficult, and the probability of recurrence is high due to chemoresistance. To overcome these defects, the development of novel therapeutic strategies is needed for more effective treatment of this serious disease.

Apoptosis (programmed cell death), plays a fundamental role in the normal development and differentiation of multicellular organisms. Apoptosis also occurs as a reaction to protect cells damaged by diseases or noxious agents. The two types of apoptosis include an extrinsic pathway that involves transmembrane death, receptor-mediated interactions and an intrinsic pathway that involves mitochondria-mediated stimuli $(4,5)$. The death receptor pathway begins with the ligation of cell surface death receptors and the activation of caspase- 8 , which activates the downstream effector caspases $(-3,-6$, and/or -7$)$. The mitochondrion-mediated pathway begins with the disruption of the mitochondrial membrane potential (MMP, $\Delta \Psi m$ ) and release of apoptogenic proteins such as cytochrome $c$ into the cytosol. Once in the cytosol, cytochrome $c$ can activate caspase-9, which in turn cleaves and activates the executioner caspases. Following activation of effector caspases such as caspase-3, cleavage of several specific substrates occurs, including poly(ADP-ribose) 
polymerase (PARP), eventually leading to apoptosis $(6,7)$. In some cells, caspase- 8 also mediates the intrinsic pathway via cleavage of the pro-apoptotic $\mathrm{Bid}$, a BH3-only protein $(8,9)$.

The caspase-cascade signaling system is also regulated by a number of different molecules, such as proteins from the Bcl-2 and the inhibitor of apoptosis protein (IAP) families. The Bcl-2 family, which has both anti-apoptotic (Bcl-2 and Bcl-xL) and pro-apoptotic (Bax, Bak, and Bid) members, acts on the mitochondrion to prevent or to facilitate the release of apoptogenic factors $(10,11)$. The IAP family proteins, on the other hand, are all endogenous inhibitors of apoptosis, and are able to bind and inhibit caspases $(12,13)$.

Reactive oxygen species (ROS) include highly reactive hydroxyl radicals $(\mathrm{OH} \cdot)$, superoxide anions $\left(\mathrm{O}_{2}^{-}\right)$, singlet oxygen $\left({ }^{1} \mathrm{O}_{2}\right)$, and hydrogen peroxide $\left(\mathrm{H}_{2} \mathrm{O}_{2}\right)$, which form as natural byproducts of the normal cellular metabolism of oxygen (14). Although ROS have important roles as intracellular messengers in cell signaling, extended high levels of ROS can cause severe damage to DNA, RNA, and proteins, eventually leading to cell death via either apoptotic or necrotic mechanisms $(15,16)$. ROS induced by a diverse range of stimuli are also produced inside organelles such as mitochondria $(17,18)$. During oxidative stressinduced cell death, ROS can target the mitochondrial membrane potential $(16,18)$.

DNA methylation is critically involved in embryonic development, chromatin structure, genomic imprinting, and chromosome inactivation and stability $(19,20)$. Aberrant hypermethylation represses transcription by way of $\mathrm{CpG}$ islands in the promoter region and is associated with gene inactivation. Decitabine (5-aza-2'-deoxycytidine) is a synthesized cytosine analogue that incorporates itself into the DNA strands of proliferating cells (21). This compound effectively inhibits DNA methylation and increases re-expression-silenced genes by covalently binding to the promoter regions of DNA methyltransferase $(22,23)$. Recently, the therapeutic activity of decitabine in acute leukemias has been tested in phase clinical trials for its excellent capability to reactivate the expression of several methylated genes (24-26). Furthermore, there are also reports on the effectiveness of decitabine for the treatment of solid tumors $(27,28)$, the connection between decitabine's clinical activity and hypomethylation activity remains unclear. In addition, other mechanisms that are independent of hypomethylation are also important for its anticancer activity, inducing cell cycle arrest, differentiation, and apoptosis, and inhibiting invasion. For example, at low concentrations, decitabine acts as an $\mathrm{S}$-phase-specific inducer, causing de novo DNA hypermethylation and silencing of transcription process in some cancer cells (29-31). However, DNA damage caused by a high concentration of decitabine induces apoptosis in a caspase-dependent or independent manner (30,32-35). While well known as a therapeutic agent due to its unique properties, the functional mechanisms by which decitabine induces antileukemic activity remain to be investigated.

In the present study, we demonstrated that decitabine induced several characteristic apoptotic symptoms in human leukemia cell lines U937 and HL60, including DNA fragmentation, chromatin condensation, activation of caspases, cleavage of PARP, and mitochondrial depolarization. We also showed that ROS are potential causes of the caspase activation that leads to decitabine-induced apoptosis.

\section{Materials and methods}

Cell culture and MTT assay. Human leukemia U937 and HL60 cells were purchased from the American Type Culture Collection (Rockville, MD) and cultured in RPMI-1640 medium (Invitrogen Corp., Carlsbad, CA) supplemented with 10\% (v/v) fetal bovine serum (FBS, Gibco BRL, Grand Island, NY), 1 mM L-glutamine, $100 \mathrm{U} / \mathrm{ml}$ penicillin, and $100 \mathrm{mg} / \mathrm{ml}$ streptomycin at $37^{\circ} \mathrm{C}$ in a humidified atmosphere of $95 \%$ air and $5 \% \mathrm{CO}_{2}$. Decitabine (Fig. 1A) was purchased from Sigma-Aldrich Chemical Co. (St. Louis, MO), dissolved in $100 \%$ dimethyl sulfoxide (DMSO) to a stock concentration $10 \mathrm{mM}$, and stored at $-80^{\circ} \mathrm{C}$. For the cell viability study, the cells were seeded onto 6 -well plates at a concentration of $5 \times 10^{4}$ cells/well, grown to $70 \%$ confluence, and then treated with various concentrations of decitabine for the indicated times. Following treatment, cell viability was determined using the 3-(4,5-dimethylthiazol-2-yl)2,5-diphenyl-tetrazolium bromide (MTT, Sigma-Aldrich) assay, which is based on the conversion of MTT to MTT-formazan by mitochondrial enzymes.

Nuclear staining with DAPI. For nuclear staining, cells were washed with phosphate-buffered saline (PBS) and fixed with $3.7 \%$ paraformaldehyde (Sigma-Aldrich) in PBS for $10 \mathrm{~min}$ at room temperature. Fixed cells were washed with PBS and stained with $2.5 \mu \mathrm{g} / \mathrm{ml}$ 4,6-diamidino-2-phenylindole (DAPI, Sigma-Aldrich) solution for $10 \mathrm{~min}$ at room temperature. Cells were then washed twice with PBS and analyzed using a fluorescence microscope (Carl Zeiss, Germany).

Agarose gel electrophoresis for DNA fragmentation assay. Following decitabine treatment, cells were lysed in a buffer containing $10 \mathrm{mM}$ Tris- $\mathrm{HCl}, \mathrm{pH} 7.4,150 \mathrm{mM} \mathrm{NaCl}, 5 \mathrm{mM}$ EDTA, and $0.5 \%$ Triton $\mathrm{X}-100$ for $1 \mathrm{~h}$ at room temperature. Lysates were vortexed and cleared by centrifugation at $19,000 \mathrm{~g}$ for $30 \mathrm{~min}$ at $4^{\circ} \mathrm{C}$. A $25: 24: 1(\mathrm{v} / \mathrm{v} / \mathrm{v})$ equal volume of neutral phenol:chloroform:isoamyl alcohol (Sigma-Aldrich) was used for extraction of DNA in the supernatant, followed by electrophoretic analysis on $1.2 \%$ agarose gels containing $0.1 \mu \mathrm{g} / \mathrm{ml}$ ethidium bromide (EtBr, Sigma-Aldrich).

Flow cytometry analysis for measurement of sub-G1 phase. The cells were harvested and washed once with PBS, fixed in ice-cold $70 \%$ ethanol, and stored at $4^{\circ} \mathrm{C}$. Prior to analysis, the cells were washed once again with PBS, suspended in $1 \mathrm{ml}$ of a cold propidium iodide (PI, Sigma-Aldrich) solution containing $100 \mu \mathrm{g} / \mathrm{ml}$ RNase A, $50 \mu \mathrm{g} / \mathrm{ml} \mathrm{PI}, 0.1 \%$ (w/v) sodium citrate, and $0.1 \%$ (v/v) NP-40, and further incubated on ice for $30 \mathrm{~min}$ in the dark. Flow cytometric analyses were carried out using a flow cytometer (FACSCalibur, Becton-Dickinson, San Jose, CA). CellQuest software was used to determine the relative DNA content, based on the presence of a red fluorescence.

Measurement of intracellular ROS and MMP $(\Delta \Psi m)$. ROS production was monitored using the stable non-polar dye 2,7 dichlorofluorescein diacetate (DCF-DA, Molecular Probes, Leiden, The Netherlands), which readily diffuses into cells (36). The cells were seeded in 24-well plates and incubated in the absence or presence of decitabine for different periods of time, after which they were incubated with $10 \mu \mathrm{M}$ DCF-DA 
for $30 \mathrm{~min}$. ROS production in the cells was monitored by flow cytometer, using CellQuest software. To measure MMP, $\Delta \Psi m$, the dual-emission potential-sensitive probe $5,5 \mathrm{~V}$, 6,6 V-tetrachloro-1,1 V,3,3 V-tetraethyl-imidacarbocyanine iodide (JC-1, Sigma-Aldrich), was used. After treatment with decitabine, $5 \times 10^{5}$ cells were collected, stained with $2 \mathrm{mg} / \mathrm{l} \mathrm{JC}-1$ at $37^{\circ} \mathrm{C}$ for $20 \mathrm{~min}$, and then analyzed with a flow cytometer (37).

Protein extraction and western blotting. The cells were harvested and lysed. The protein concentrations were measured using a Bio-Rad protein assay (Bio-Rad Laboratories, Hercules, $\mathrm{CA}$ ), according to the manufacturer's instructions. For western blot analysis, an equal amount of protein was subjected to electrophoresis on SDS-polyacrylamide gel and transferred by electroblotting to a nitrocellulose membrane (Schleicher \& Schuell, Keene, NH). The blots were probed with the desired antibodies for $1 \mathrm{~h}$, incubated with the diluted enzyme-linked secondary antibody, and visualized by enhanced chemiluminescence (ECL), according to the recommended procedure (Amersham Corp., Arlington Heights, IL) (38). The primary antibodies were purchased from Santa Cruz Biotechnology Inc. (Santa Cruz, CA) and Cell Signaling Technology, Inc. (Boston, MA). The peroxidase-labeled donkey anti-rabbit immunoglobulin and peroxidase-labeled sheep anti-mouse immunoglobulin were purchased from Amersham Corp.

In vitro caspase activity assay. Activities of caspases were determined by use of colorimetric assay kits, which utilize synthetic tetrapeptides (Asp-Glu-Val-Asp (DEAD) for caspase-3; Ile-Glu-Thr-Asp (IETD) for caspase-8; Leu-Glu-His-Asp (LEHD) for caspase-9, respectively) labeled with p-nitroaniline (pNA). Briefly, decitabine-treated and untreated cells were lysed in the supplied lysis buffer. Supernatants were collected and incubated with the supplied reaction buffer containing DTT and DEAD-pNA, IETD-pNA, or LEHD-pNA as substrates at $37^{\circ} \mathrm{C}$. The reactions were measured by changes in absorbance at $405 \mathrm{~nm}$ using the VERSAmax tunable microplate reader.

Statistical analysis. Unless otherwise indicated, each result is expressed as the mean $\pm \mathrm{SD}$ of data obtained from triplicate experiments. Statistical analysis was performed using a paired Student's t-test. Differences at $\mathrm{p}<0.05$ were considered statistically significant.

\section{Results}

Inhibition of cell viability by decitabine in human leukemia cells. To evaluate the effects of decitabine on cell viability, U937 and HL60 cells were stimulated with various concentrations of decitabine for the indicated times, and an MTT assay was performed. As shown in Fig. 1B, decitabine induced a decrease in cell viability in a concentration- and time-dependent manner. For example, treatment with $20 \mu \mathrm{M}$ decitabine for $72 \mathrm{~h}$ resulted in 58 and 60\% inhibition in U937 and HL60 cells, respectively, which was associated with many morphological changes (data not shown).

Induction of apoptosis by decitabine in human leukemia cells. In order to determine whether the decrease in cell viability in U937 and HL60 cells by decitabine treatment was due to the induction
A

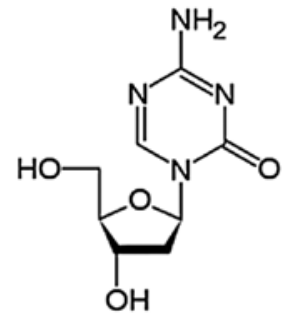

B

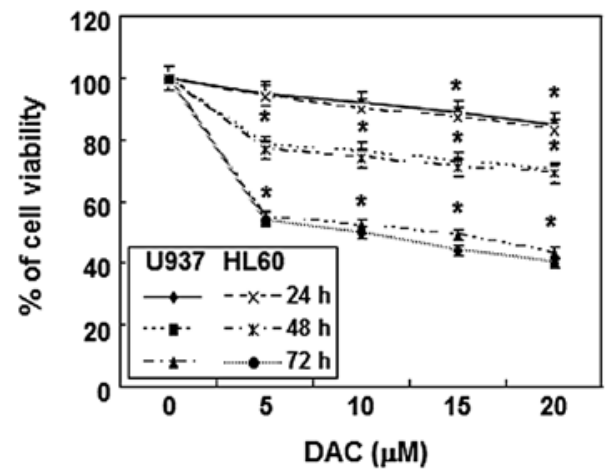

Figure 1. Inhibition of cell viability by decitabine treatment in human leukemia cells. (A) Chemical structure of decitabine. (B) U937 and HL60 cells were plated at a concentration of $2.5 \times 10^{5}$ cells per $60 \mathrm{~mm}$ plate. Following $24 \mathrm{~h}$ of stabilization, cells were treated with various concentrations of decitabine (DAC) for the indicated times. Cell viability was determined by MTT assay. Results are expressed as percentage of the vehicle treated control \pm standard deviation (SD) of three separate experiments. A Student's t-test ( $\mathrm{p}<0.05$ vs. untreated control) was used for determination of significance.

of apoptosis, three established criteria were subsequently used for the assessment of apoptosis. First, morphological changes of cells were determined using DAPI staining; as shown in Fig. 2A, treatment with decitabine resulted in observation of a significant number of cells with chromatin condensation, loss of nuclear construction, and formation of apoptotic bodies in a time-dependent fashion, whereas these features were not observed in control cells. Second, we analyzed DNA fragmentation, which is another hallmark of apoptosis. Following agarose gel electrophoresis of DNAs from cells treated with decitabine, a typical ladder pattern of internucleosomal fragmentation was observed. In contrast, DNA fragmentation was barely detected in control cells (Fig. 2B). In addition, the degree of apoptosis in cells treated with decitabine was determined using flow cytometric analysis for detection of hypodiploid cell populations. As shown in Fig. 2C, the addition of decitabine to cells resulted in increased accumulation of cells in the sub-G1 phase in a manner similar to that observed with decitabine-induced viability inhibition, the formation of apoptotic bodies, and accumulation of extranuclear fragmented DNA. This finding suggests that U937 and HL60 cells may undergo apoptosis after exposure to decitabine, and there is a good correlation between the extent of apoptosis and inhibition of growth.

Effects of decitabine on the expression of apoptosis-related proteins in human leukemia cells. The role of $\mathrm{Bcl}-2$ and the IAP family, as well as death receptor-related members in decitabine- 
A

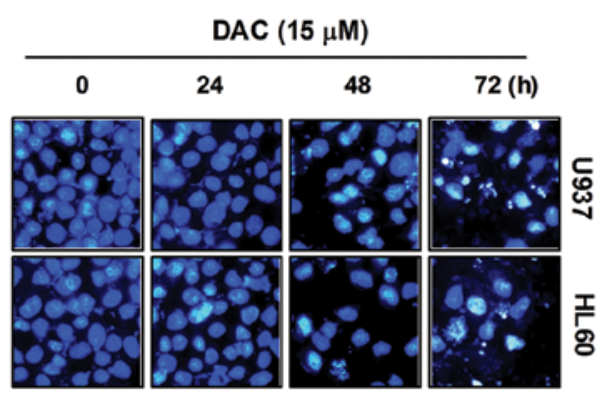

C

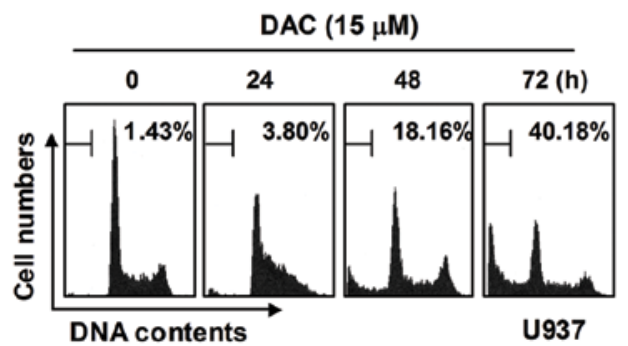

B
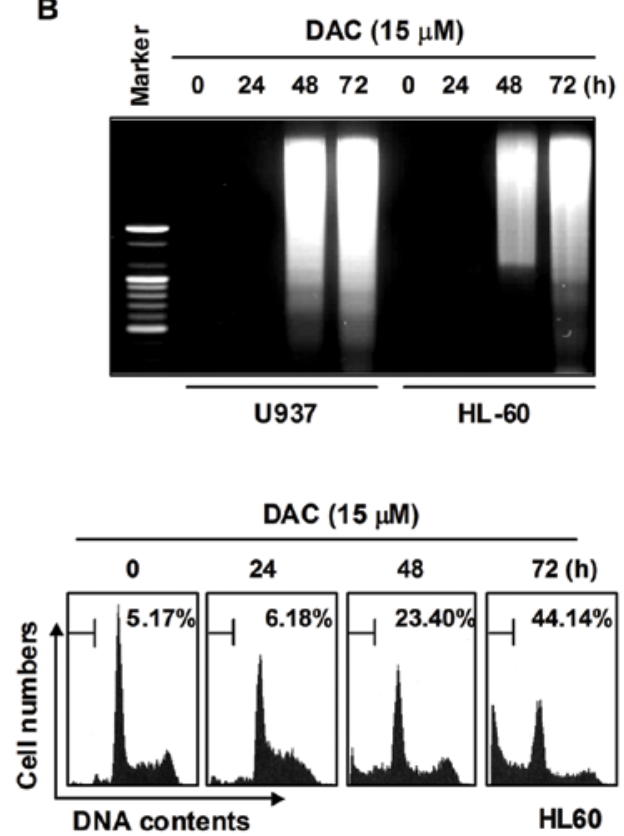

Figure 2. Induction of apoptosis by decitabine treatment in human leukemia cells. (A) Following treatment with $15 \mu \mathrm{M}$ decitabine for the indicated times, cells were fixed, stained with DAPI for $10 \mathrm{~min}$, and the nuclear morphology was then photographed under fluorescence using a blue filter. Magnification x400. (B) For analysis of DNA fragmentation, genomic DNA was extracted from cells grown under the same conditions as (A) and then electrophoresed on a $1.2 \%$ agarose gel. Marker indicates a size marker of the DNA ladder. (C) To quantify the degree of apoptosis induced by decitabine, cells were evaluated for sub-G1 DNA content, which represents the fractions undergoing apoptotic DNA degradation, using a flow cytometer. Data represent the mean of two independent experiments.

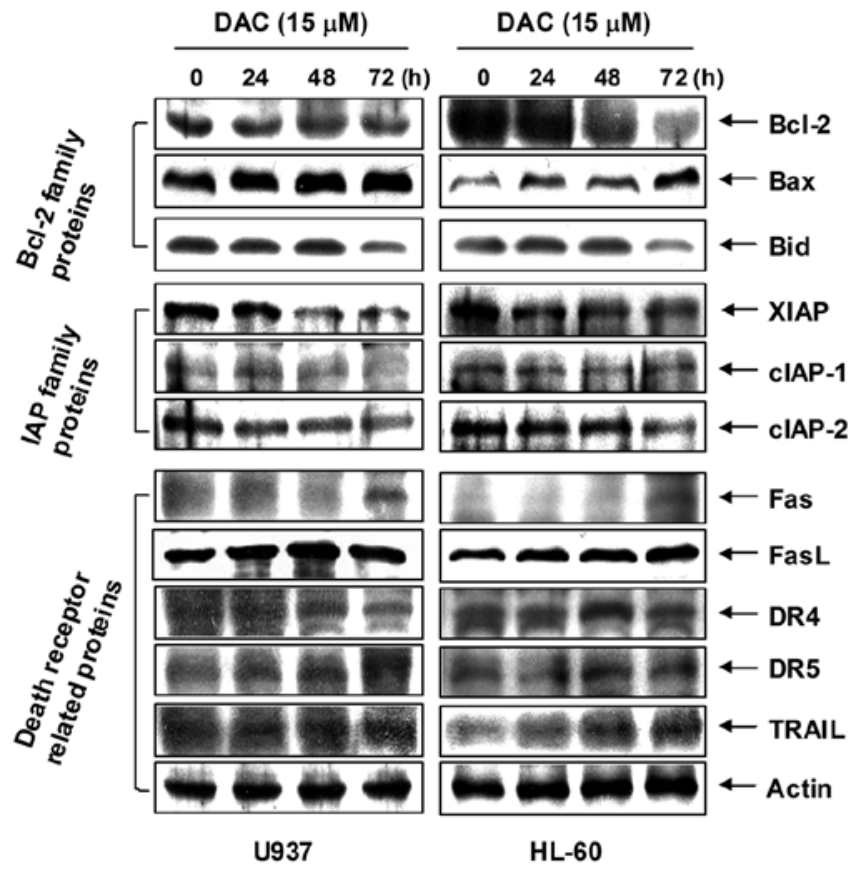

Figure 3. Effects of decitabine on levels of apoptosis-related proteins in human leukemia cells. U937 and HL60 cells were treated with $15 \mu \mathrm{M}$ decitabine for the indicated times. The cells were lysed and then equal amounts of cell lysates $(30 \mu \mathrm{g})$ were separated on SDS-polyacrylamide gels and transferred to nitrocellulose membranes. Membranes were probed with the indicated antibodies and the proteins were visualized using an ECL detection system. Actin was used as an internal control.

mediated apoptosis, was determined by western blotting for measurement of expression of their proteins. As shown in Fig. 3, the levels of anti-apoptotic Bcl-2 proteins were decreased in response to decitabine treatment; however, the levels of proapoptotic Bax were increased in a concentration-dependent manner. Under these conditions, the levels of total pro-apoptotic protein Bid, a BH3-only pro-apoptotic member of the Bcl-2 family, concentration-dependently decreased in response to decitabine treatment. In addition, the levels of anti-apoptotic IAP family proteins such as XIAP, cIAP-1, and cIAP-2 were markedly inhibited by decitabine treatment in a concentrationdependent manner. Furthermore, the results showed that decitabine treatment resulted in a concentration-dependent increase in the level of death receptor-related proteins including Fas, Fas ligand (FasL), death receptor (DR) 4, DR5, and tumor necrosis factor-related apoptosis-inducing ligand (TRAIL).

Activation of caspases by decitabine in human leukemia cells. Expression levels and activities of caspase-3, -8 and -9 in U937 and HL60 cells exposed to decitabine were measured in order to determine whether decitabine-induced apoptosis is associated with the activation of caspases. As shown in Fig. 4A, immunoblotting results showed that decitabine treatment induced a concentration-dependent increase in levels of active-caspase-3, -8 and -9 proteins. For further quantification of the proteolytic activation of caspases, protein in the lysates of cells treated with decitabine was normalized and then assayed for in vitro activities using fluorogenic substrates. As shown in Fig. 4B, treatment with decitabine resulted in a significant concentration-dependent increase of the activities of caspase- $3,-8$ and -9 compared with control cells. In addition, decitabine treatment led to progressive proteolytic cleavage of PARP, a well-known substrate protein of activated caspase-3. 
A



B
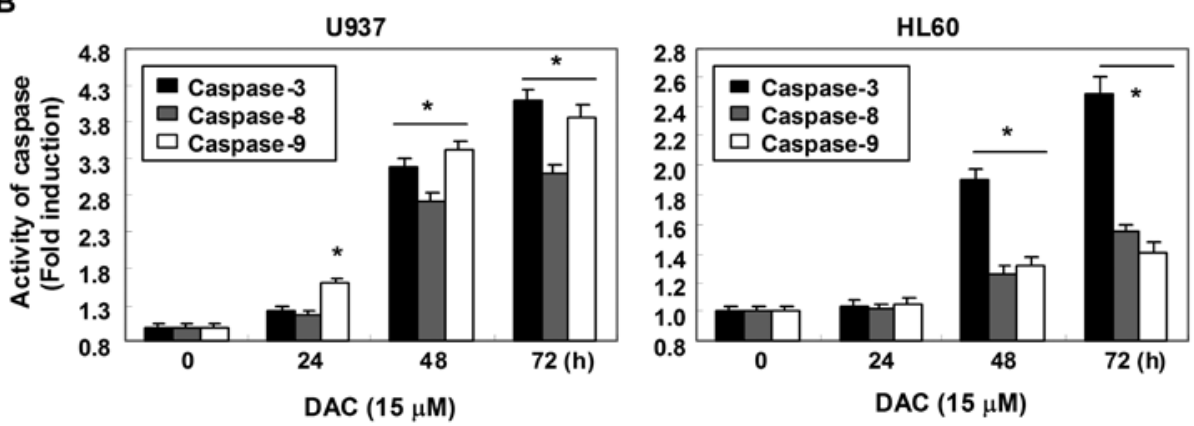

Figure 4. Activation of caspases and degradation of PARP proteins by decitabine treatment in human leukemia cells. (A) Cells were treated with $15 \mu \mathrm{M}$ decitabine for the indicated times, lysed, and then equal amounts of cell lysates (30 $\mu \mathrm{g}$ ) were separated on SDS-polyacrylamide gels and transferred to nitrocellulose membranes. Membranes were probed with the indicated antibodies. An ECL detection system was used for visualization of the proteins. Actin was used as an internal control. (B) Cells grown under the same conditions as (A) were collected and lysed. Aliquots were incubated with DEVD-pNA, IETD-pNA, and LEHD-pNA for caspase-3, -8 and -9, respectively, as substrates at the indicated time-points. Released fluorescence products were measured. Each point represents the mean $\pm \mathrm{SD}$ of representative experiments performed at least three times. A Student's t-test ( $\mathrm{p}<0.05$ vs. untreated control) was used for analysis of statistical significance of the results.

In order to demonstrate that the activation of caspases is a key step in the apoptotic pathway induced by decitabine, U937 and HL60 cells were pretreated with potential caspase-specific inhibitors (z-DEVD-fmk, z-IETD-fmk, and z-LEHD-fmk for the inactivation of caspase- $3,-8$ and -9 , respectively) for $1 \mathrm{~h}$, followed by treatment with decitabine for $72 \mathrm{~h}$. As shown in Fig. 5, pretreatment with caspase inhibitors significantly attenuated chromatin condensation and the formation of apoptotic bodies, and restored the decreased viability. These results indicate that decitabine treatment induces apoptosis in U937 and HL60 cells through a caspase-dependent pathway.

Loss of MMP values and increase of ROS generation by decitabine in human leukemia cells. To examine the role of mitochondria in apoptosis induced by decitabine, we attempted to characterize the relationship between changes in the MMP and ROS production. For this study, the effects of decitabine on the levels of MMP were monitored via a flow cytometer using the mitochondrial-specific probe, JC-1. As shown in Fig. 6A, the loss of MMP values showed a time-dependent increase by decitabine treatment, indicating that decitabine induced mitochondrial membrane hyperpolarization by depolarization. Next, ROS production was measured using a cell-permeant, oxidation-sensitive dye, DCF-DA. In U937 cells exposed to decitabine for the indicated time periods, generation of ROS was observed at $0.5 \mathrm{~h}$ and the levels continued to increase at $3 \mathrm{~h}$ (Fig. 6B). A similar trend in ROS generation was also observed in HL60 cells in response to decitabine treatment. As expected, the ROS scavenger N-acetyl-L-cysteine (NAC), a commonly used reactive oxygen intermediate scavenger, markedly blocked the levels of ROS from the decitabine-treated U937 and HL60 cells at $10 \mathrm{mM}$.

Decitabine-induced apoptosis is associated with the generation of ROS in human leukemia cells. In order to show that generation of ROS is a key step in the decitabine-induced apoptotic pathway, cells were pretreated with $10 \mathrm{mM}$ NAC for $1 \mathrm{~h}$, followed by treatment with decitabine for $72 \mathrm{~h}$. As shown in Fig. 7A-C, blocking of ROS generation by pretreatment of cells with NAC effectively prevented decitabine-induced downregulation of pro-caspase-3 and XIAP expression, cleavage of PARP activation of caspase-3, and loss of MMP. In addition, pretreatment of cells with NAC also prevented decitabine-induced chromatin condensation (Fig. 7D), which was associated with recovered cell viability (Fig.7E). Collectively, these findings suggest that an increase in ROS generation is required for the occurrence of decitabine-induced apoptosis in U937 and HL60 cells. 
A

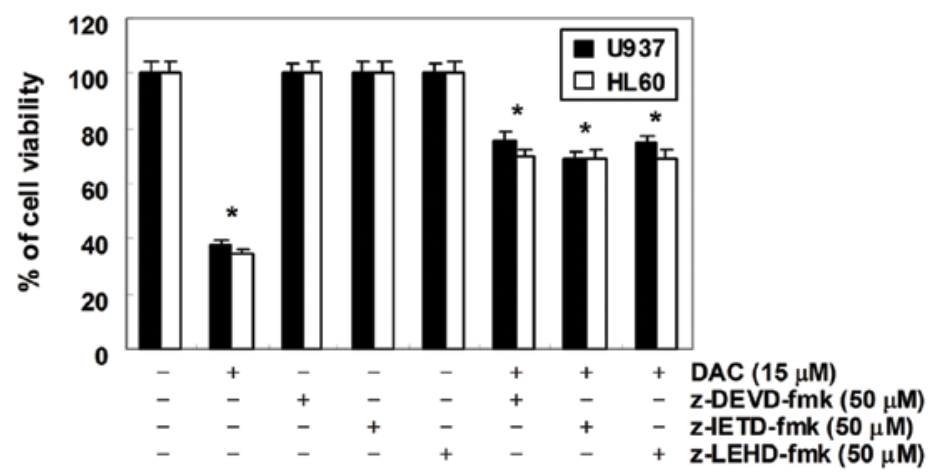

B

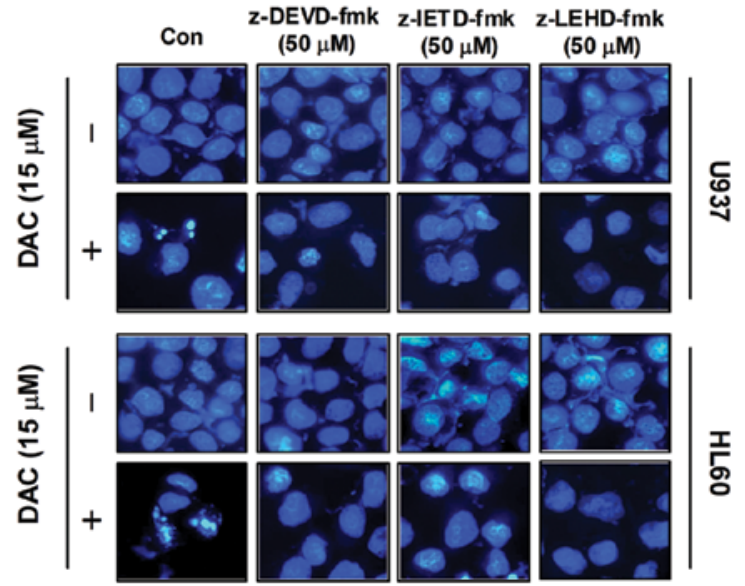

Figure 5. Inhibition of decitabine-induced apoptosis by caspase inhibitors in human leukemia cells. (A) Cells were pretreated for $1 \mathrm{~h}$ with or without the indicated caspase inhibitors (50 $\mu \mathrm{M}$; z-DEVD-fmk, caspase-3 inhibitor; z-IETD-fmk, caspase-8 inhibitor; z-LEHD-fmk, caspase-9 inhibitor; z-VAD-fmk, pan-caspase inhibitor) and then treated with $15 \mu \mathrm{M}$ decitabine for an additional $72 \mathrm{~h}$. The degree of growth inhibition was determined by MTT assay. Results are expressed as percentage of the vehicle treated control $\pm \mathrm{SD}$ of three separate experiments. A Student's t-test ( $\mathrm{p}<0.05$ vs. untreated control or agent alone) was used for determination of significance. (B) Cells grown under the same conditions as (A) were fixed, stained with DAPI for 10 min, and photographed with a fluorescence microscope using a blue filter. Magnification x 400 .

A

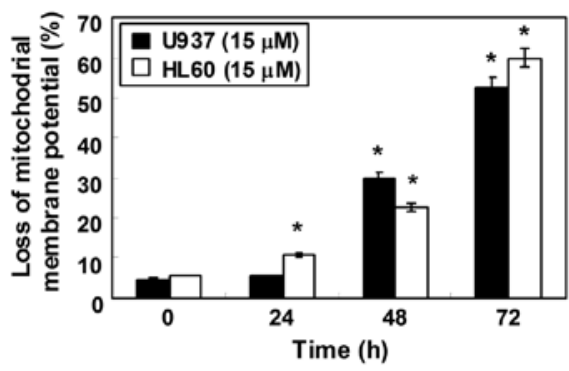

B
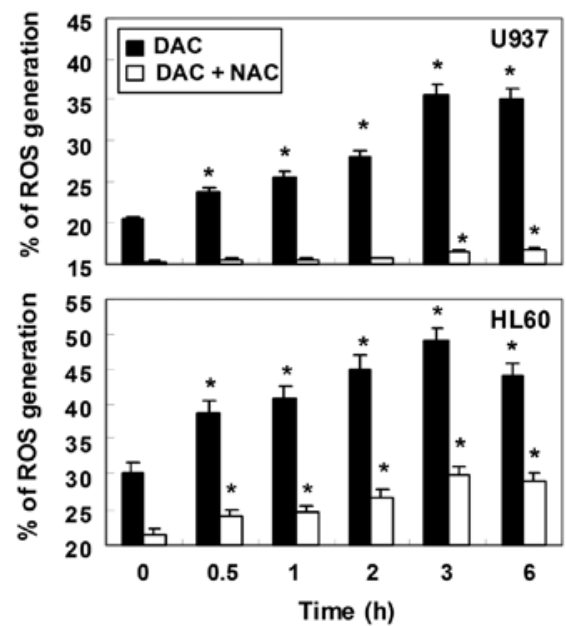

\section{Discussion}

Although an increasing amount of data indicate that decitabine, a well-known demethylating agent in cancer therapy, can suppress the growth of cultured cancer cells by causing cell cycle arrest and apoptosis induction (29-35), the signaling pathways involved in apoptosis induction by this compound are poorly defined. In the present study, we demonstrated that decitabine-induced anti-proliferative effects in human leukemia U937 and HL60 cells were related to the induction of apoptosis, as confirmed by measurement of chromatin condensation of nuclei, DNA fragmentation, and the induction of the sub-G1 phase. Our data also demonstrated that decitabine induced apoptosis of U937 and HL60 cells through generation of ROS and mitochondrial

Figure 6. Loss of MMP and generation of ROS by decitabine treatment in human leukemia cells. (A) Cells were treated with $15 \mu \mathrm{M}$ decitabine for the indicated times and stained with JC-1 for $20 \mathrm{~min}$ at $37^{\circ} \mathrm{C}$. The mean JC-1 fluorescence intensity was then detected using a flow cytometer. (B) Cells were treated with or without NAC $(10 \mathrm{mM})$ for $1 \mathrm{~h}$ before challenge with $15 \mu \mathrm{M}$ decitabine for the indicated times. The cells were incubated with $10 \mu \mathrm{M} \mathrm{DCF}-\mathrm{DA}$ at $37^{\circ} \mathrm{C}$ for $20 \mathrm{~min}$, and ROS generation was measured using a flow cytometer. Results are expressed as percentage of the vehicle treated control \pm SD of three separate experiments. A Student's t-test ( $\mathrm{p}<<0.05$ vs. untreated control) was used for determination of significance. 
A

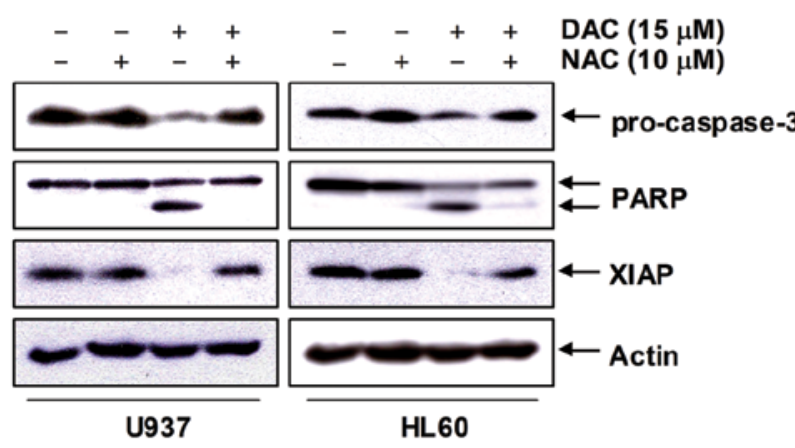

D

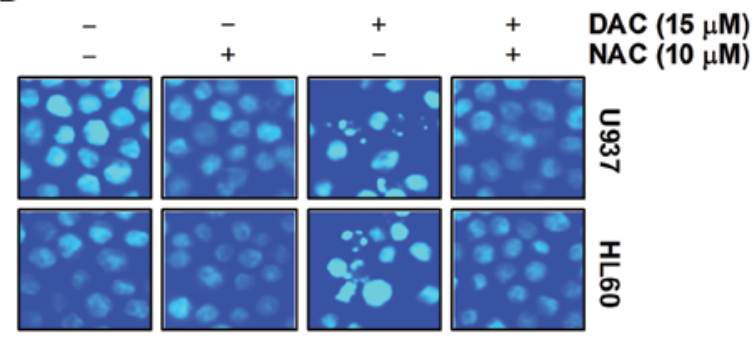

B

C

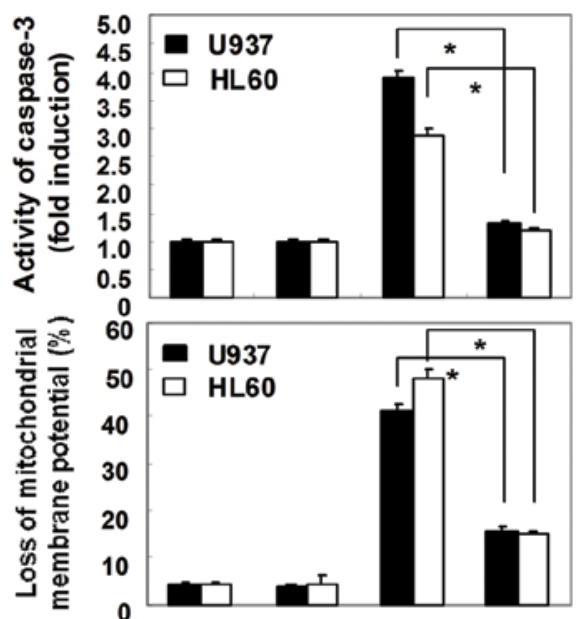

E

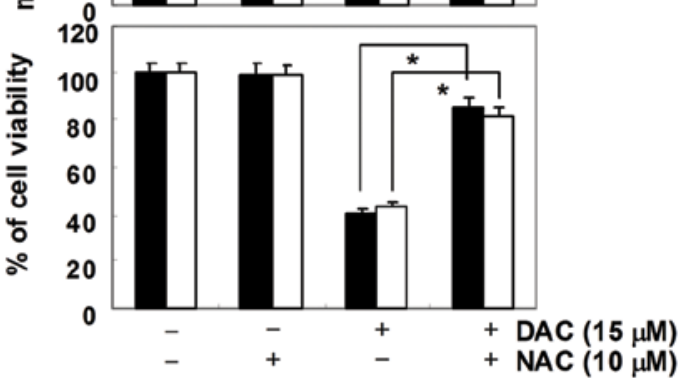

Figure 7. Decitabine-induced apoptosis is associated with ROS generation in human leukemia cells. Cells were incubated with $15 \mu \mathrm{M}$ decitabine for $72 \mathrm{~h}$ after pretreatment with or without $10 \mathrm{mM}$ NAC. The levels of caspase-3, PARP, and XIAP proteins were determined using western blot analysis (A). Cells were evaluated for DAPI staining using a fluorescent microscope. Magnification x400 (D). Caspase-3 activity was determined using ELISA (B), loss of MMP was determined using a flow cytometer $(\mathrm{C})$, and cell viability was determined using the MTT assay (E). Data are expressed as overall means \pm SD from three independent experiments. A Student's t-test ( $\mathrm{p}<0.05$ vs. decitabine alone) was used for determination of significance.

dysfunction, suggesting that ROS act as upstream signaling molecules for the initiation of cell death.

Apoptosis plays an important role in the normal development and differentiation of multicellular organisms, which are characterized by morphological and biological changes such as cytoplasmic shrinkage, chromatin condensation, and DNA degradation. Apoptosis also serves as a critical protective mechanism against carcinogenesis caused by mutations of genetic materials of normal cells or various carcinogens (39). A variety of stimuli can trigger it, including death receptor-mediated signaling (extrinsic pathway) or intracellular stresses (intrinsic pathway) $(4,5)$. Our data indicated that decitabine-induced apoptosis of U937 and HL60 cells was associated with increased enzymatic activity of both the extrinsic and intrinsic caspase cascades, such as caspase-8 and -9 (Fig. 4). Although the truncated Bid form was not detected, the levels of intact Bid proteins were gradually downregulated in a concentration-dependent manner by decitabine in both cell lines (Fig. 3). Decitabine treatment also decreased the levels of IAP family proteins, such as XIAP, cIAP-1, and cIAP-2, and the anti-apoptotic Bcl-2, whereas the levels of pro-apoptotic Bax and death receptor-related proteins, including Fas, FasL, DR4, DR5, and TRAIL, were markedly increased in response to decitabine treatment (Fig. 3). Additionally, decitabine caused a significant reduction in the MMP values, which was connected with the activation of caspase- 3 and the concomitant degradation of PARP. Thus, the results indicated that caspase- 8 activation by decitabine triggered mitochondrial apoptotic events by inducing conformational changes in apoptotic proteins. Furthermore, treatment with decitabine in the presence of caspase inhibitors was found to significantly prevent apoptosis (Fig. 5). Therefore, the data suggest that decitabine-induced apoptosis in both the U937 and HL60 cell lines is caspase-dependent, and the apoptotic effects of decitabine appear to involve activation of both the intrinsic and extrinsic pathways.

In addition to apoptosis, ROS are known to mediate other intracellular signaling cascades. Oxidative stress is generally considered to be an important regulator of apoptosis $(15,17)$. Many studies have suggested that a disproportionate production of ROS leads to oxidative stress, dysfunction of cell organelles like the mitochondria, and eventually apoptosis or necrosis (16). Recently, ROS-mediated caspase activation and mitochondrial dysfunction have been suggested as critical for decitabineinduced apoptosis in several cancer cell lines $(35,40,41)$; however, the current role of mitochondrial functional changes associated with ROS generation in the response of human leukemia cells to decitabine has not yet been well explored. Therefore, we next investigated the question of whether apoptosis by decitabine in U937 and HL60 cells was associated with the generation of ROS.

We found a significant early overproduction of ROS in decitabine-treated U937 and HL60 cells; furthermore, co-culture with NAC, a commonly used ROS scavenger, effectively blocked this ROS generation (Fig. 6). The present results revealed that the activation of caspase- 3 and degradation of PARP proteins in 
decitabine-treated cells were ROS-dependent, and that co-culture with NAC effectively blocked decitabine-induced growth inhibition and apoptosis in U937 as well as HL60 cells. The findings from the present study also indicated that the loss of MMP in decitabine-treated cells is ROS-dependent, which suggests that ROS may act upstream of caspase activation. In addition, blocking of ROS generation prevented decitabine-induced downregulation of XIAP. Because IAP family proteins are also substrates of activated caspase-3 $(42,43)$, the observed decrease in XIAP expression may be due to caspase-3-mediated processing following decitabine treatment. Since ROS have the potential to induce the collapse of the MMP, and consequently trigger the series of events leading to the mitochondria-associated apoptotic pathway $(15,44)$, our findings suggest the involvement of ROS production and mitochondrial dysfunction in decitabine-induced caspase-mediated apoptosis in U937 and HL60 cells.

In conclusion, our study demonstrated that human leukemia cells undergo apoptosis in response to treatment with decitabine, and that this occurs through a mitochondria-mediated pathway that requires ROS generation upstream for disruption of the MMP, which leads to subsequent activation of caspases. The present data emphasize the key role of ROS in apoptosis induced by decitabine in human leukemia cells, and indicate that a positive correlation exists between ROS and mitochondrial events leading to apoptosis, and may aid in understanding of the mechanisms for the anticancer activity of human leukemia. Taken together, although further investigation of its activity in vivo is necessary to elaborate and exploit this promise, these results suggest that human leukemia may be a potential chemotherapeutic agent for the treatment of leukemia patients.

\section{Acknowledgements}

This study was supported by Basic Science Research Program through the National Research Foundation of Korea (NRF) funded by the Ministry of Education, Science, and Technology (2010-0008843 and 2010-001730).

\section{References}

1. Chang H, Lin H, Yi L, Zhu J, Zhou Y, Mi M and Zhang Q: 3,6-Dihydroxyflavone induces apoptosis in leukemia HL-60 cells via reactive oxygen species-mediated $\mathrm{p} 38$ MAPK/JNK pathway. Eur J Pharmacol 648: 31-38, 2010.

2. Abramson N and Melton B: Leukocytosis: basics of clinical assessment. Am Fam Physician 62: 2053-2060, 2000.

3. Gilliland DG, Jordan CT and Felix CA: The molecular basis of leukemia. Hematology Am Soc Hematol Educ Program: 80-97, 2004.

4. Green DR and Reed JC: Mitochondria and apoptosis. Science 281: 1309-1312, 1998.

5. Siegelin MD, Habel A and Gaiser T: 17-AAG sensitized malignant glioma cells to death-receptor mediated apoptosis. Neurobiol Dis 33: 243-249, 2009.

6. Lazebnik YA, Kaufmann SH, Desnoyers S, Poirier GG and Earnshaw WC: Cleavage of poly(ADP-ribose) polymerase by a proteinase with properties like ICE. Nature 371: 346-347, 1994.

7. Jin $\mathrm{Z}$ and El-Deiry WS: Overview of cell death signaling pathways. Cancer Biol Ther 4: 139-163, 2005.

8. Li H, Zhu H, Xu CJ and Yuan J: Cleavage of BID by caspase 8 mediates the mitochondrial damage in the Fas pathway of apoptosis Cell 94: 491-501, 1998.

9. Luo X, Budihardjo I, Zou H, Slaughter C and Wang X: Bid, a bcl-2 interacting protein, mediates cytochrome c release from mitochondria in response to activation of cell surface death receptors. Cell 94: 481-490, 1998.
10. Dlugosz PJ, Billen LP, Annis MG, Zhu W, Zhang Z, Lin J, Leber B and Andrews DW: Bcl-2 changes conformation to inhibit Bax oligomerization. EMBO J 25: 2287-2296, 2006.

11. Park C, Jin CY, Kwon HJ, Hwang HJ, Kim GY, Choi IW, Kwon TK, Kim BW, Kim WJ and Choi YH: Induction of apoptosis by esculetin in human leukemia U937 cells: roles of Bcl-2 and extracellularregulated kinase signaling. Toxicol In Vitro 24: 486-494, 2010.

12. De Laurenzi V and Melino G: Apoptosis. The little devil of death. Nature 406: 135-136, 2000.

13. Gao Z, Tian Y, Wang J, Yin Q, Wu H, Li YM and Jiang X: A dimeric Smac/diablo peptide directly relieves caspase-3 inhibition by XIAP. Dynamic and cooperative regulation of XIAP by Smac/Diablo. J Biol Chem 282: 30718-30727, 2007.

14. Crack PJ and Taylor JM: Reactive oxygen species and the modulation of stroke. Free Radic Boil Med 38: 1433-1444, 2005.

15. Fiers W, Beyaert R, Declercq W and Vandenabeele P: More than one way to die: apoptosis, necrosis and reactive oxygen damage. Oncogene 18: 7719-7730, 1999.

16. Skulachev VP: Bioenergetic aspects of apoptosis, necrosis and mitoptosis. Apoptosis 11: 473-485, 2006.

17. Chakraborti T, Das S, Mondal M, Roychoudhury S and Chakraborti S: Oxidant, mitochondria and calcium: an overview. Cell Signal 11: 77-85, 1999.

18. Choi WY, Kim GY, Lee WH and Choi YH: Sanguinarine, a benzophenanthridine alkaloid, induces apoptosis in MDA-MB-231 human breast carcinoma cells through a reactive oxygen speciesmediated mitochondrial pathway. Chemotherapy 54: 279-287, 2008.

19. Robertson KD: DNA methylation and human disease. Nat Rev Genet 6: 597-610, 2005.

20. Watanabe Y and Maekawa M: Methylation of DNA in cancer. Adv Clin Chem 52: 145-167, 2010.

21. Jones PA and Taylor SM: Cellular differentiation, cytidine analogs and DNA methylation. Cell 20: 85-93, 1980.

22. Bender CM, Pao MM and Jones PA: Inhibition of DNA methylation by 5-aza-2'-deoxycytidine suppresses the growth of human tumor cell lines. Cancer Res 58: 95-101, 1998.

23. Karpf AR and Jones DA: Reactivating the expression of methylation silenced genes in human cancer. Oncogene 21: 5496-5503, 2002.

24. Kihslinger JE and Godley LA: The use of hypomethylating agents in the treatment of hematologic malignancies. Leuk Lymphoma 48: 1676-1695, 2007.

25. Santos FP, Kantarjian H, Garcia-Manero G, Issa JP and Ravandi F: Decitabine in the treatment of myelodysplastic syndromes. Expert Rev Anticancer Ther 10: 9-22, 2010.

26. Robak T: New nucleoside analogs for patients with hematological malignancies. Expert Opin Investig Drugs 20: 343-359, 2011.

27. Issa JP and Kantarjian HM: Targeting DNA methylation. Clin Cancer Res 15: 3938-3946, 2009.

28. Boumber Y and Issa JP: Epigenetics in cancer: what's the future? Oncology 25: 220-206, 2011.

29. Hurtubise A and Momparler RL: Effect of histone deacetylase inhibitorLAQ824 on antineoplasticaction of 5-Aza-2'-deoxycytidine (decitabine) on human breast carcinoma cells. Cancer Chemother Pharmacol 58: 618-625, 2006.

30. Valdez BC, Li Y, Murray D, Corn P, Champlin RE and Andersson BS: 5-Aza-2'-deoxycytidine sensitizes busulfanresistant myeloid leukemia cells by regulating expression of genes involved in cell cycle checkpoint and apoptosis. Leuk Res 34: 364-372, 2010.

31. Ng KP, Ebrahem Q, Negrotto S, Mahfouz RZ, Link KA, Hu Z, Gu X, Advani A, Kalaycio M, Sobecks R, et al: p53 independent epigenetic-differentiation treatment in xenotransplant models of acute myeloid leukemia. Leukemia 25: 1739-1750, 2011.

32. Festuccia C, Gravina GL, D'Alessandro AM, Millimaggi D, Di Rocco C, Dolo V, Ricevuto E, Vicentini C and Bologna M: Downmodulation of dimethyl transferase activity enhances tumor necrosis factor-related apoptosis-inducing ligand-induced apoptosis in prostate cancer cells. Int J Oncol 33: 381-388, 2008.

33. Deng T and Zhang Y: 5-Aza-2'-deoxycytidine reactivates expression of RUNX3 by deletion of DNA methyltransferases leading to caspase independent apoptosis in colorectal cancer Lovo cells. Biomed Pharmacother 63: 492-500, 2009.

34. Capper D, Gaiser T, Hartmann C, Habel A, Mueller W, HeroldMende C, von Deimling A and Siegelin MD: Stem-cell-like glioma cells are resistant to TRAIL/Apo2L and exhibit down-regulation of caspase- 8 by promoter methylation. Acta Neuropathol 117: 445-456, 2009. 
35. Ruiz-Magaña MJ, Rodríguez-Vargas JM, Morales JC, Saldivia MA, Schulze-Osthoff K and Ruiz-Ruiz C: The DNA methyltransferase inhibitors zebularine and decitabine induce mitochondria-mediated apoptosis and DNA damage in p53 mutant leukemic T cells. Int J Cancer 130: 1195-1207, 2012

36. Kim SY, Kang HT, Choi HR and Park SC: Biliverdin reductase A in the prevention of cellular senescence against oxidative stress. Exp Mol Med 43: 15-23, 2011.

37. Choi JH, Choi AY, Yoon H, Choe W, Yoon KS, Ha J, Yeo EJ and Kang I: Baicalein protects HT22 murine hippocampal neuronal cells against endoplasmic reticulum stress-induced apoptosis through inhibition of reactive oxygen species production and CHOP induction. Exp Mol Med 42: 811-822, 2010.

38. Jeong JH, Ryu DS, Suk DH and Lee DS: Anti-inflammatory effects of ethanol extract from Orostachys japonicus on modulation of signal pathways in LPS-stimulated RAW 264.7 cells. BMB Rep 44: 399-404, 2011.

39. Hengartner MO: The biochemistry of apoptosis. Nature 407: $770-776,2000$
40. Lin CT, Lin WH, Lee KD and Tzeng PY: DNA mismatch repair as an effector for promoting phorbol ester-induced apoptotic DNA damage and cell killing: implications in tumor promotion. Int J Cancer 119: 1776-1784, 2006.

41. Brodská B and Holoubek A: Generation of reactive oxygen species during apoptosis induced by DNA-damaging agents and/ or histone deacetylase inhibitors. Oxid Med Cell Longev 2011: 253529, 2011.

42. Hell K, Saleh M, Crescenzo GD, O'Connor-McCourt MD and Nicholson DW: Substrate cleavage by caspases generates protein fragments with Smac/Diablo-like activities. Cell Death Differ 10: 1234-1239, 2003.

43. Choi YE, Butterworth M, Malladi S, Duckett CS, Cohen GM and Bratton SB: The E3 ubiquitin ligase cIAP1 binds and ubiquitinates caspase- 3 and -7 via unique mechanisms at distinct steps in their processing. J Biol Chem 284: 12772-12782, 2009.

44. Fleury C, Mignotte B and Vayssière JL: Mitochondrial reactive oxygen species in cell death signaling. Biochimie 84: 131-141, 2002 . 